TITLE:

\title{
Nonconvex perturbations of maximal monotone differential inclusions
}

AUTHOR(S):

Staicu, Vasile

CITATION:

Staicu, Vasile. Nonconvex perturbations of maximal monotone differential inclusions. 数 理解析研究所講究録 1998, 1061: 95-107

ISSUE DATE:

1998-08

URL:

http://hdl.handle.net/2433/62386

RIGHT: 


\title{
Nonconvex perturbations of maximal monotone differential inclusions*
}

\author{
Vasile Staicu \\ Department of Mathematics \\ Aveiro University \\ 3810 Aveiro - Portugal \\ e-mail: vasile@mat.ua.pt
}

\begin{abstract}
We survey some results concerning existence and qualitative properties of solutions to differential inclusions of the form $\dot{x} \in-A x+F(t, x)$, where $A$ is an maximal monotone operator and $F$ is a multifunction with closed nonempty and nonconvex values.
\end{abstract}

\section{Introduction.}

The theory of nonlinear evolution equations has been the subject of many studies made in the last years (see [3], [14], [20], [21], [32], [28], [30], and the references therein).

Y. Kômura [22] introduced in 1967 the notions of maximal monotone operator and of nonlinear semigroup of contractions, in Hilbert spaces, and later in 1971, Brézis [13] found out that the family of subdifferential operators form a special subclass of maximal monotone operators, generating a nonlinear semigroup with smoothing effect. Thereafter, the Kômura-Brézis theory have been developed by many people in various directions.

A broad range of interesting problems in Partial Differential Equations is covered by initial value problems of the form

$$
\dot{x} \in-A x+f(t), x(0)=x_{0}
$$

where $A$ is a maximal monotone operator and $f$ is a integrable function (see [3], [14]). Existence of solutions to (1) follows, to some extent, from the basic relation

*Presented at the RIMS Symposium on nonlinear evolution equations, kyoto, Japan, October 20-23, 1997 


$$
\frac{d}{d t}\|x(t)\|^{2}=2\langle\dot{x}(t), x(t)\rangle
$$

that applied to two solutions of (1), by the monotonicity of $A$ and the minus sign on the right hand side, yields that their distance is nonincreasing. This reasonings allows the construction of a Cauchy sequence of approximate solutions, converging to a solution. The existence of the right approximate solutions is supplied by the maximality of $A$, that permits the use of the Yoshida approximations. Therefore existence is a result of completeness, of maximality and of having the sign minus at the right hand side.

The same conditions and the Kakutani-Ky Fan fixed point theorem are used in [2] to prove existence for

$$
\dot{x} \in-A x+F(t, x), x(0)=x_{0}
$$

where the single-valued perturbation was replaced by an upper semicontinuous convexvalued multifunction. Such differential inclusions, arise in the study of many problems with practical interest like the synthesis of optimal control, reaction-diffusion systems, differential games, etc., and was studied by many authors (see [32], [14], [23],[6], mainly for the case when $F$ is convex-valued).

Although the case when the multifunction $F$ is convex-valued was more studied, the case when $F$ has not convex values seems to be more realistic (see [4], p. 208), and it was tackled first by Cellina and Marchi [15], for continuous and compact-valued $F$. They used a fixed point approach based on a continuous selection argument introduced by Antosiewicz and Cellina in [1], where the case $A=0$ was considered.

Subsequently this argument was generalized by Fryszkowski [18], which proved the existence of a continuous selection from lower semicontinuous multifunctions with decomposable values.

Existence of solutions to (2) for lower semicontinuous $F$ was proved by Colombo, Fonda and Ornelas [17], and by Mitidieri and Vrabie [25], in finite and respectively, infinite dimensional spaces. Both in [17] and in [25] solutions are obtained as fixed points of a suitable compact transformation, relying on the Fryszkowski selection theorem for lower semicontinuous multifunctions with decomposable values [18].

Another approach consists in finding a selection $f$ of the multifunction $F$, and solving the problem

$$
\dot{x} \in-A x+f(t, x), x(0)=x_{0} .
$$

Since the values of $F$ are not assumed convex, no continuous selection exists, in general. Yet, as we proved in the joint paper with A. Bressan [12] a "directionally continuous" selection $f$ from $F$ exists such that for each initial value the problem (3) admits a solution. This method of "directionally continuous selections", introduced by Bressan [8] for the case $A=0$, permits to prove qualitative properties of the solution set of (2) (see [12]). 
For the case when the perturbation $F$ is upper semicontinuous cyclically monotone we refer to [27] and [16]. In [27] the multifunction $F$ takes covex values in a Hilbert space ( $F$ is a subdiferential), while in the joint paper with Cellina [16], $F$ takes nonconvex values in a finite dimensional space. Some extensions to infinite dimensional spaces of [16] were obtained in [31] (see also [6] and [5]).

After some preliminaries in Section 2, we will explain in Section 3 how the fixed point approach is used to prove existence to the Cauchy problem (2), where the perturbation $F$ is lower semicontinuous with no convex values. In Section 4 we present the method of directionaly cntinuous selections and illustrate how the study of solutions to (2) with lower semicontinuous and nonconvex values perturbation $F$ reduces such a study for the case when the perturbation is upper semicontinuous and convex valued.

\section{Preliminaries.}

Let $\left(X, d_{X}\right)$ and $\left(Y, d_{Y}\right)$ be two metric spaces, $2^{Y}$ be the family of all nonempty subsets of $Y$ and for any two closed subsets $A$ and $B \in 2^{Y}$ let

$$
h(A, B)=\max \left\{\sup _{a \in A} \inf _{b \in B} d_{Y}(a, b), \sup _{b \in B} \inf _{a \in A} d_{Y}(a, b),\right\}
$$

be the Pompeiu-Hausdorff pseudo-distance from $A$ to $B$.

A multifunction $F: X \rightarrow 2^{Y}$ is said to be:

(i) lower semicontinuous (l.s.c.) on $X$ if for every $x_{0} \in X$ and for each open set $V$ such that $V \cap F\left(x_{0}\right) \neq \emptyset$ there exists a neighborhood $U$ of $x_{0}$ such that $V \cap F(x) \neq \emptyset$ for each $x \in U$.

(ii) upper semicontinuous (u.s.c) on $X$ if for every $x_{0} \in X$ and for each open set $V$ such that $F\left(x_{0}\right) \subset V$ there exists a neighborhood $U$ of $x_{0}$ such that $F\left(x_{0}\right) \subset V$ for each $x \in U$.

(iii) Hausdorff continuous on $X$ if for every $x_{0} \in X$ and for every $\varepsilon>0$ there exists $\delta>0$ such that $d_{X}\left(x, x_{0}\right)<\delta$ implies $h\left(F(x), F\left(x_{0}\right)\right)<\varepsilon$.

Remark 1 (i) $F$ is l.s.c. on $X$ iff for every closed subset $C$ of $Y$ the set $\{x \in X: F(x) \subset C\}$ is closed in $X$.

(ii) $F$ is u.s.c. on $X$ iff for every closed subset $C$ of $Y$ the $\operatorname{set}\{x \in X: F(x) \cap C \neq \emptyset\}$ is closed in $X$.

(iii) if $F$ is compact valued then $F$ is Hausdorff continuous iff it is both upper and lower semicontinuous.

If $\mathcal{F}$ is a $\sigma$-algebra of measurable subsets on $X$, then we say that the multifunction $F: X \rightarrow 2^{Y}$ is $\mathcal{F}$-measurable if $\{x \in X: F(x) \cap C \neq \emptyset\} \in \mathcal{F}$ for every closed set $C \subset Y$.

By selection from the multifunction $F: X \rightarrow 2^{Y}$ we mean any function $f: X \rightarrow Y$ such that $f(x) \in F(x)$, for every $x \in X$. 
Let now $(T, \mathcal{F}, \mu)$ be a finite, positive, nonatomic measure space, $Y$ be Banach space with norm $\|.\|_{Y}$. We denote by $L^{1}(T, Y)$ the Banach space of Bochner integrable functions $u: T \rightarrow Y$ with norm $\|u\|_{1}=\int_{T}\|u(t)\|_{Y} d \mu$. if

Following Hiai and Umegaki[19] we say that a subset $K \subset L^{1}(T, Y)$ is decomposable

$$
u \chi_{A}+v \chi_{T \backslash A} \in K \text {, whenever } u, v \in K, A \in \mathcal{F} \text {. }
$$

Olech [26] pointed out that many results concerning decomposable sets can be obtained from famous statements concerning convex sets, just substituting the word "convex" with the word "decomposable".

The well known Michael theorem [24] states that a lower semicontinuous multifunction from a paracompact space $X$ into closed convex subsets of a linear space $Y$ admits a continuous selection. Convexity assumption is there essential.

An analog of this theorem to lower semicontinuous multifunctions with decomposable values in $L^{1}(T, Y)$ was obtained by Fryskowski [18]:

Theorem 1 [18]. If $X$ is a compact space and $\Phi: X \rightarrow 2^{L^{1}(T, Y)}$ is lower semicontinuous with closed and decomposable values then $\Phi$ admits a continuous selection.

Remark that Antosiewicz and Cellina [1] proved this theorem when $X$ is a compact set of continuous functions, $\Phi(x)=\{u: u(t) \in F(t, x(t))$ a.e. $\}$ and $F$ is a multifunction continuous with closed and not necessarily convex values.

Bressan and Colombo [10] extend the Fryskowski's results to lower semicontinuous multifunction with closed decomposable values defined on a separable space.

Let $X$ be a Banach space with norm $\|\cdot\|, X^{*}$ be the dual space of $X$ with norm $\|\cdot\|_{*}$ and let $H: X \rightarrow 2^{X^{*}}$ be the duality map given by

$$
H(x)=\left\{x^{*} \in X^{*}: x^{*}(x)=\|x\|^{2}=\left\|x^{*}\right\|_{*}^{2}\right\} .
$$

Then we consider on $X$ the semi-inner product $\langle\cdot, \cdot\rangle_{+}$defined by

$$
\langle y, x\rangle_{+}=\sup \left\{x^{*}(y): x^{*} \in H(x)\right\}
$$

For $A \subset X \times X$ and $x \in X$ we set $A x=\{y \in X:(x, y) \in A\}$ and denote by $D(A)$ and $R(A)$ the domain and the range of $A$ defined by

$$
D(A)=\{x \in X: A x \neq \emptyset\}, R(A)=\bigcup_{x \in D(A)} A x .
$$

We say that $A$ is $m$-accretive if the following two conditions are satisfied:

(i) $\left\langle y^{\prime}-y, x^{\prime}-x\right\rangle_{+} \geq 0$, whenever $(x, y),\left(x^{\prime}, y^{\prime}\right) \in A$,

(ii) $R(I+t A)=X$, for all $t>0$, where $I$ is the identity map on $X$. 
Remark that if $X$ is a Hilbert space with scalar product $\langle.,$.$\rangle then A$ is m-accretive in $X$ iff it is maximal monotone, that is, it satisfies $(i)$ and $(i i)$ for $\langle.,$.$\rangle instead of \langle., .\rangle_{+}$.

Let $A \subset X \times X$ be m-accretive, and let $I$ be one of the intervals $[0, T]$ or $[0, \infty[$. For $f \in L^{1}(I, X)$ and $x_{0} \in c l(D(A))$, consider the initial value problem:

$$
\dot{x} \in-A x+f(t), x(0)=x_{0} .
$$

We call strong solution of $\left(P_{f}\right)$ on $I$ any continuos function $x: I \rightarrow c l(D(A))$ with derivative $\dot{x} \in L_{l o c}^{1}(I, X)$ such that $x(0)=x_{0}$ and $\dot{x}(t) \in A x(t)+f(t)$ a.e. $t \in I$.

Following [7] we call integral solution of $\left(P_{f}\right)$ on $[0, T]$. any continuous function $x$ : $[0, T] \rightarrow \operatorname{cl}(D(A))$ with $x(0)=x_{0}$ and such that, for every $(\bar{x}, \bar{y}) \in A$ and $0 \leq s \leq t \leq T$,

$$
\|x(t)-\bar{x}\|^{2} \leq\|x(s)-\bar{x}\|^{2}+2 \int_{s}^{t}\langle f(\tau)+\bar{y}, x(\tau)-\bar{x}\rangle_{+} d \tau
$$

Remark that any strong solution is also integral solution

Let $F: I \times X \rightarrow 2^{X}$ be a multifunction and for $x_{0} \in c l(D(A))$, consider the initial value problem :

$$
\dot{x} \in A x+F(t, x), x(0)=x_{0} .
$$

A continuous function $x: I \rightarrow c l(D(A))$ is called strong solution (resp. integral solution) of the problem $\left(P_{F}\right)$ if there exists $f \in L^{1}(I, X)$ such that $f(t) \in F(t, x(t))$ a.e. in $I$ and $x$ is a strong solution (resp. integral solution) of the corresponding initial problem $\left(P_{f}\right)$

\section{The fixed point approach.}

We will consider the initial-value problem

$$
\dot{x} \in-A x+F(t, x), x(0)=x_{0}
$$

and distinguish two cases:

(i) $A$ is a maximal monotone operator in $\mathbb{R}^{n}$ and $F:[0, \infty) \times \mathbb{R}^{n} \rightarrow 2^{\mathbb{R}^{n}}$ is lower semicontinuous, compact-valued multifunction.

(ii) $A$ is a m-accretive operator in a Banach space $X$ and $F:[0, T] \times X \rightarrow 2^{X}$ is lower semicontinuous closed-valued multifunction.

We show how the fixed point approach is used to prove: existence of a strong solution on $[0, \infty)$ in the first case and a integral solution on $[0, T]$ in second one.

Let assume $A$ is maximal monotone in $\mathbb{R}^{n}$ and $x_{0} \in c l(D(A))$. Recall that for any $f \in L_{\text {loc }}^{1}\left([0, \infty), \mathbb{R}^{n}\right)$ there exists a unique strong solution $x^{f}:[0, \infty) \rightarrow \mathbb{R}^{n}$ to the Cauchy problem

$$
\dot{x} \in-A x+f(t), x(0)=x_{0}
$$


that is $x^{f}$ is continuos on $[0, \infty)$, absolutely continuous on every compact subset of $(0, \infty)$, and satisfies $x^{f}(0)=x_{0}$, and for a.e. $t \in[0, \infty)$ :

$$
x^{f}(t) \in D(A) \text { and } x^{f}(t) \in-A x(t)+f(t) .
$$

Moreover, for every $t \geq 0$

$$
\left\|x_{f}(t)-x_{g}(t)\right\| \leq \int_{0}^{t}\|f(s)-g(s)\| d s,
$$

hence the map $i: L_{\text {loc }}^{1}\left([0, \infty), \mathbb{R}^{n}\right) \rightarrow L_{\text {loc }}^{1}\left([0, \infty), \mathbb{R}^{n}\right)$ defined by $i(f)=. x^{f}$ is continuous.

Let $F:[0, \infty) \times \mathbb{R}^{n} \rightarrow 2^{\mathbb{R}^{n}}$ be given. A continuous function $x:[0, \infty) \rightarrow \operatorname{cl}(D(A))$ is called a strong solution to the problem $\left(P_{F}\right)$ if it is a strong solution to $\left(P_{f}\right)$ for some $f \in L_{\text {loc }}^{1}\left([0, \infty), \mathbb{R}^{n}\right)$ such that $f(t) \in F(t, x(t))$ a.e.

Let $\mathcal{L} \times \mathcal{B}$ be the $\sigma$-algebra on $[0, \infty) \times \operatorname{cl}(D(A))$ generated by the products $L \times B$, where $L \subset[0, \infty)$ is Lebesgue measurable and $B \subset \operatorname{cl}(D(A))$ is a Borel subset

Assume that $F:[0, \infty) \times \mathbb{R}^{n} \rightarrow 2^{\mathbb{R}^{n}}$ satisfies the following assumptions:

$\left(\mathrm{H}_{1}\right) F$ is $\mathcal{L} \times \mathcal{B}$-measurable,

$\left(\mathrm{H}_{2}\right)$ for each $t \geq 0, F(t,$.$) is lower semicontinuous.$

$\left(\mathrm{H}_{3}\right)$ there exists two non-negative locally integrable functions $\alpha, \beta:[0, \infty) \rightarrow \mathbb{R}$ such that for every $(t, x) \in[0, \infty) \times \operatorname{cl}(D(A))$ :

$$
\sup \{\|y\|: y \in F(t, x)\} \leq \alpha(t)\|x\|+\beta(t) .
$$

The following result was proved in [17]:

Theorem 2 If $A$ is a maximal monotone operator in $\mathbb{R}^{n}$ and $F$ is a compact-valued multifunction satisfying $\left(H_{1}\right)-\left(H_{3}\right)$ then for any $x_{0} \in \operatorname{cl}(D(A))$ there exists $x:[0, \infty) \rightarrow$ $\mathbb{R}^{n}$, a strong solution to the problem $\left(P_{F}\right)$.

Let give a sketch of the proof in order to show how the fixed point approach is used.

Define like in [15], an appropriate nonempty compact and convex set $K \subset L_{\text {loc }}^{1}\left([0, \infty), \mathbb{R}^{n}\right)$, (as the closure in $L_{\text {loc }}^{1}\left([0, \infty), \mathbb{R}^{n}\right)$ of the set of all absolutely continuous functions $v$ with $v(0)=x_{0}, v(t) \in c l(D(A))$ and satisfying some appropriate inequalities) and construct a continuous map $g: K \rightarrow L_{l o c}^{1}\left([0, \infty), \mathbb{R}^{n}\right)$ such that, $g(x)(t) \in F(t, x(t))$. Then the map $s: K \rightarrow L_{\text {loc }}^{1}\left([0, \infty), \mathbb{R}^{n}\right)$ defined by $s(x)=i(g(x))$ is continuous and satisfies $s(K) \subset K$, hence by the Schauder-Tichonov theorem it admits a fixed point which is a solution of the problem $\left(P_{F}\right)$.

Let show how the selection $g$ is constructed. For $n=1,2, \ldots$, set $I_{n}=[0, n]$ and $K_{n}=\left\{\left.v\right|_{I_{n}}: v \in K\right\} \subset L^{1}\left(I_{n}, \mathbb{R}^{n}\right)$.

Construct then a sequence of continuous maps $g_{n}: K_{n} \rightarrow L^{1}\left(I_{n}, \mathbb{R}^{n}\right)$ satisfying the following two conditions, for any $x \in K_{n}$ :

(a) $g_{n}(x)(t) \in F(t, x(t))$ for a. e. $t \in I_{n}$,

(b) if $n>1, g_{n}(x)(t)=g_{n-1}(x)(t)$, for a.e. $t \in I_{n-1}$. 
Since $K_{1}$ is compact and the multifunction $G_{1}: K_{1} \rightarrow 2^{L^{1}\left(I_{1}, R^{n}\right)}$ defined by

$$
G_{1}(x)=\left\{u \in L^{1}\left(I_{1}, \mathbb{R}^{n}\right): u(t) \in F(t, x(t)) \text { a. e. in } I_{1}\right\}
$$

is lower semicontinuous with closed nonempty decomposable values, by Fryszkowski's theorem, there exists $g_{1}: K_{1} \rightarrow L^{1}\left(I_{1}, \mathbb{R}^{n}\right)$, a continuous selection from $G_{1}$

Let $n>2$ and assume that $g_{n-1}$ was already constructed, satisfying $(a)$ and $(b)$. The multifunction $G_{n}$ defined by

$$
\begin{gathered}
G_{n}(x)=\left\{u \in L^{1}\left(I_{n}, \mathbb{R}^{n}\right): u(t) \in F(t, x(t)) \text { a. e. in } I_{n} \text { and } .\right. \\
\left.u(t)=g_{n-1}(x)(t), \text { a.e in } I_{n-1}\right\}
\end{gathered}
$$

is lower semicontinuous with closed nonempty decomposable values. Then by Fryszkowski's theorem, there exists a continuos map $g_{n}: K_{n} \rightarrow L^{1}\left(I_{n}, \mathbb{R}^{n}\right)$ such that $(a)$ and $(b)$ are satisfyed.

Define then $g: K \rightarrow L_{l o c}^{1}\left([0, \infty), \mathbb{R}^{n}\right)$. by setting

$$
\left.g(x)\right|_{I_{n}}=g_{n}(x) .
$$

Then $g$ is continuous, well defined and $g(x)(t) \in F(t, x(t))$

Let $s: K \rightarrow L_{\text {loc }}^{1}\left([0, \infty), \mathbb{R}^{n}\right)$ be defined by $s(x)=i(g(x))=x^{g(x)}$ is continuous and $s(K) \subset K$. Then a fixed point of of $s$ exists by the Schauder-Tichonov theorem and it is a solution of the problem $\left(P_{F}\right)$.

An infinite dimensional analog of the result from above obtained by Mitidieri and Vrabie [25] is the following:

Theorem 3 Assume that $A$ is a m-accretive operator in a separable Banach space $X$ such that $-A$ generates a compact semigroup $\{S(t): t \geq 0\}, F:[0, T] \times X \rightarrow 2^{X}$ is a lower semicontinuous nonempty and closed valued multifunction such that for each bounded set $B \subset \operatorname{cl}(D(A))$ there exists $h \in L^{1}([0, T], \mathbb{R})$ with $\sup \{|F(t, x)|: x \in B\} \leq h(t)$ a.e. $t \in(0, T)$, where $|F(t, x)|:=\sup \{\|v\|: v \in F(t, x)\}$.

Then for every $x_{0} \in \operatorname{cl}(D(A))$ there exists $T_{0}=T\left(x_{0}\right)$ such that the problem $\left(P_{F}\right)$ has at least one integral solution on $\left[0, T_{0}\right]$.

The proof of this result that we sketch in the following also use the fixed point approach.

For $x_{0} \in \operatorname{cl}(D(A))$ and $r>0$ fixed choose $T_{0} \in(0, T]$ such that:

$$
\sup \left\{|F(t, x)|:\left\|x-x_{0}\right\| \leq r \leq h(t) \text { a.e. in }\left(0, T_{0}\right)\right\}
$$

and

$$
\left\|S(t) x_{0}-x_{0}\right\|+\int_{0}^{t} h(s) d s \leq r, \text { a.e. in }\left(0, T_{0}\right) .
$$


Define

$$
G=\left\{g \in L^{1}\left(\left[0, T_{0}\right], X\right):\|g(t)\| \leq h(t) \text { a.e. in }\left(0, T_{0}\right)\right\}
$$

and for $g \in G$ let $x^{g}$ be the unique integral solution of the problem

$$
\dot{x} \in-A x+g(t), x(0)=x_{0} .
$$

Since the semigroup generated by $-A$ is compact and $G$ is uniformly integrable it follows that the closure in the space $C\left(\left[0, T_{0}\right], X\right)$ of the set $\left\{x^{g}: g \in G\right\}$, denoted by $K$ is compact in that space.

The multifunction $\mathcal{K}: K \rightarrow 2^{L^{1}\left(\left[0, T_{0}\right], X\right)}$ defined by

$$
\mathcal{K}(x)=\left\{f \in L^{1}\left(\left[0, T_{0}\right], X\right): f(t) \in F(t, x(t)) \text { a.e. in }\left(0, T_{0}\right)\right\}
$$

is lower semicontinuous with closed nonempty and decomposable values, hence by Fryszkowski's theorem there exists a continuous function $f: K \rightarrow L^{1}\left(\left[0, T_{0}\right], X\right)$ such that $f(x)(t) \in$ $F(t, x(t))$ a.e. in $\left(0, T_{0}\right)$,for each $x \in K$.

Moreover, since $f$ is continuous and $K$ is compact, by Mazur's theorem it follows that the closed convex hull of $f(K)$ denoted by $K_{O}$ is compact and convex.

Since the map $\varphi: K \rightarrow L^{1}\left(\left[0, T_{0}\right], X\right)$ defined by $\varphi(g)=f\left(x^{g}\right)$ is continuous from the compact and convex set $K_{O}$ into itself by Schauder fixed point theorem there exists $g \in K_{O}$ such that $\varphi(g)=g$.

Therefore, a integral solution of the problem $\left(P_{F}\right)$ is precisely the integral solution $x^{g}$ of the problem $\left(P_{g}\right)$ with $g=f\left(x^{g}\right)$

Remark 2 The next section will contain a new approach introduced in [12], which not only provides a conceptually simpler construction of solutions of $\left(P_{F}\right)$ but it also yield new qualitative results on the solution set, which apparently cannot be obtained by the techniques presented in this section.

\section{The method of directionally continuous selections.}

In all this section we assume that $X$ is a Banach space, $A$ is a m-accretive operator in $X$ with domain $D(A),\{S(t): t \geq 0\}$ is the semigroup of contractions on $\operatorname{cl}(D(A))$ generated by $-A$. and denote by $\Omega=\mathbb{R} \times \operatorname{cl}(D(A))$.

Let $F:[0, T] \times X \rightarrow 2^{X}$ be a bounded lower semicontinuous multifunction and for $x_{0} \in \operatorname{cl}(D(A))$, consider the initial value problem :

$$
\dot{x} \in A x+F(t, x), x(0)=x_{0} .
$$

The method of directionally continuous selections consists in finding a selection $f$ of the multifunction $F$, and solving the problem

$$
\dot{x} \in-A x+f(t, x), x(0)=x_{0} .
$$


Since the values of $F$ are not assumed convex, no continuous selection exists, in general. Yet, we will construct a new topology $\mathcal{T}^{+}$on $\Omega$, stronger than the usual metric one, such that a selection from $F$ continuous with respect to this topology exists such that for each initial value the problem (4) admits a solution. Moreover, using this selection we construct an upper semicontinuous multifunction with convex values $G$ such that every integral solution of the problem

$$
\dot{x} \in-A x+G(t, x), x(0)=x_{0} .
$$

is also an integral solution of the problem $\left(P_{F}\right)$. In this way we reduce the study of the solutions of $\left(P_{F}\right)$ to the (somewhat easier) one of the solutions of $\left(P_{G}\right)$.

In order to construct the topology $\mathcal{T}^{+}$on $\Omega$ let fix $M>0$ and for every $(t, x) \in \Omega$ and $\varepsilon>0$ define like in [12]:

$V(t, x, \varepsilon)=\left\{\begin{array}{c}(s, y) \in \Omega: t \leq s<t+\varepsilon,\|y-S(\tau) x\| \leq M(s-t), \\ \text { for some } \tau \in[0, s-t]\end{array}\right\}$

Remark that $V(t, x, \varepsilon)$ is generated by all the trajectories of thecontrol system

$$
\dot{y}(s) \in-A y(s) u_{1}(s)+M u_{2}(s), y(t)=x, s \in[t, t+\varepsilon),
$$

and

Theorem 4 The family of sets $\{V(t, x, \varepsilon):(t, x) \in \Omega, \varepsilon>0\}$ is a basis of open neighborhoods for a topology $\mathcal{T}^{+}$on $\Omega$, stronger than the metric one, and each set $V(t, x, \varepsilon)$ is closed-open in the topology $\mathcal{T}^{+}$.

We say that a multifunction $F: \Omega \rightarrow 2^{X}$ is Scorza-Dragoni lower semicontinuous (resp. Scorza-Dragoni upper semicontinuous) if there exists a sequence of disjoint compact sets $J_{\nu} \subset[0, T]$ with meas $\left([0, T] \backslash \bigcup_{\nu} J_{\nu}\right)=0$ such that $F$ is lower semicontinuous (resp. upper semicontinuous) restricted to each set $\Omega_{\nu}=\left\{(t, x) \in \Omega: t \in J_{\nu}\right\}$.

The notion of Scorza-Dragoni $\mathcal{T}^{+}$-continuous selection is defined in a similar way.

The following result was proved in [12]:

Theorem 5 Let $A$ be m-accretive such that $-A$ generates the semigroup $\{S(t): t \geq 0\}, \Omega \subset$ $\mathbb{R} \times \operatorname{cl}(D(A))$ be locally compact and for a fixed $M>0$ let $\mathcal{T}^{+}$be the topology from above. Then every Scorza-Dragoni lower semicontinuous multifunction $F: \Omega \rightarrow 2^{X}$ admits a Scorza-Dragoni $\mathcal{T}^{+}$-continuous selection.

In order to obtain this result by the selection theorem in [11] it sufices to prove that every $(t, x) \in \Omega$ has a countable basis of neighborhoods, in the standard topology, consisting of sets which are closed-open in the topology $\mathcal{T}^{+}$

Such sets are given by

$$
W_{t, x}^{n}=V\left(t-\frac{1}{n}, x, \frac{2}{n}\right), n \geq 1 .
$$


Since is Scorza-Dragoni lower semicontinuous there exists a sequence of disjoint compact sets $J_{\nu} \subset[0, T]$ with meas $\left([0, T] \backslash \bigcup_{\nu} J_{\nu}\right)=0$ such that $F$ is lower semicontinuous (resp. upper semicontinuous) restricted to each set $\Omega_{\nu}=\left\{(t, x) \in \Omega: t \in J_{\nu}\right\}$.

For each $\nu \geq 1$ we obtain then by Theorem 1 in [11] a $\mathcal{T}^{+}$-continuous selection $f_{\nu}$ from $F$ restricted to $\Omega_{\nu}$.

Now, for $\varphi$ an arbitrary selection from $F$, the function defined by

$$
f(t, x)= \begin{cases}f_{\nu}(t, x), & t \in J_{\nu} \\ \varphi(t, x), & t \notin \cup J_{\nu}\end{cases}
$$

is a Scorza-Dragoni $\mathcal{T}^{+}$-continuous selection from $F$.

This selection result implies the following

Theorem 6 Let $X$ be a reflexive Banach space, $A$ be a m-accretive operator in $X, \Omega \subset$ $[0, T] \times c l(D(A))$ be locally compact and $F: \Omega \rightarrow 2^{X}$ be a bounded Scorza-Dragoni lower semicontinuous multifunction with closed nonempty values. Then there exists a ScorzaDragoni upper semicontinuous multifunction $G: \Omega \rightarrow 2^{X}$ with closed, convex, nonempty values such that every integral solution of

$$
\dot{x} \in-A x+G(t, x), x(0)=x_{0} .
$$

is also an integral solution of

$$
\dot{x} \in-A x+F(t, x), x(0)=x_{0} .
$$

Since $F$ is bounded there exists $L>0$ such that $F(t, x) \subset B(0, L)$, for all $(t, x) \in \Omega$. For $M>L$ let $f: \Omega \rightarrow X$ be the Scorza-Dragoni $\mathcal{T}^{+}$-continuous selection given by the preceding theorem. For $(t, x) \in \Omega_{\nu}$ let

$$
G_{\nu}(t, x)=\bigcap_{\varepsilon>0} \overline{c o}\left\{f(s, y):(s, y) \in \Omega_{\nu},|s-t|<\varepsilon,\|y-x\|<\varepsilon\right\} .
$$

Then the multifunction defined by

$$
G(t, x)= \begin{cases}G_{\nu}(t, x), & t \in J_{\nu} \\ \{f(t, x)\}, & t \notin \cup J_{\nu}\end{cases}
$$

satisfies the conclusion of the theorem.

Remark 3 The preceding result permits to obtain qualitative properties of solutions of $\left(P_{F}\right)$, for lower semicontinuous nonconvex valued $F$ as corolaries of similar properties of solutions of $\left(P_{G}\right)$ for the upper semicontinuous convex-valued $G$. Using this patern we proved in [12] the connectedness of the solution set of $\left(P_{F}\right)$.

Remark 4 The compactness assumption requested by the selection theorem in [11] is avoided in [29], where a general selection theorem in [9] is used instead of the one in [11]. 


\section{References}

[1] H. A. Antosiewicz and A. Cellina. Continuous selections and differential relations. J. Differential Equations, 19:386-398, 1975.

[2] H. Attouch and D. Damlamian. On multivalued evolution equations in hilbert spaces. Israel J. Math., 12:373-390, 1972.

[3] V. Barbu. Nonlinear semigroups and differential equations in Banach spaces. Nordhoff International Publ., 1976.

[4] V. Barbu. Optimal control of variational inequalities. Pitman, 1984. Reasearch Notes in Mathematics 100.

[5] H. Benabdellah, C. Castaign, and A. Salvadori. Compactness and discretization methods for differential inclusions and evolution problems. Atti Sem. Mat. Fis. Univ. Modena, XLV:9-51, 1997.

[6] H. Benabdellah and A. Faik. Perturbations convexes et non convexes des equations d'evolution. Port. Math., 53:187-208, 1996.

[7] P. Benillan. Solutions integrales dïequations d'evolution dans un espace de banach. C. R. Acad. Sci. Paris, 279:47-50, 1972.

[8] A. Bressan. Directionally continuous selections and differential inclusions. Funkcial. Ekvac., 31:459-470, 1988.

[9] A. Bressan and G. Colombo. Selections and representations of multifunctions in paracompact spaces. Studia Math., 102:987-992, 1989.

[10] A. Bressan and G. Colombo. Extensions and selections of maps with decomposable values. Studia Math., 102:209-216, 1992.

[11] A. Bressan and A. Cortesi. Directionally continuous selections in banach spaces. Nonlinear Analysis, 13:987-992, 1989.

[12] A. Bressan and V. Staicu. On nonconvex perturbations of maximal monotone differential inclusions. Set-Valued Analysis, 2:415-437, 1994.

[13] H. Brezis. Proprietes regularizantes de certain semi-groupes non linĆaires. Israel J. Math., 9:513-534, 1971.

[14] H. Brezis. Operateurs maximaux monotones et semigroupes de contractions dans les espaces de Hilbert. North-Holand, Amsterdam, 1973.

[15] A. Cellina and M. Marchi. Non-convex perturbations of maximal monotone differential inclusions. Israel J. Math., 46:1-11, 1983. 
[16] A. Cellina and V. Staicu. On evolution equations having montonicities of opposite sign. J. Differential Equations, 90:71-80, 1991.

[17] G. Colombo, A. Fonda, and A. Ornelas. Lower semicontinuous perturbations of maximal monotone differential inclusions. Israel J. Math., 61:211-218, 1988.

[18] A. Fryszkowski. Continuous selections for a class of nonconvex multivalued maps. Studia Math., 76:163-174, 1981.

[19] F. Hiai and H. Umegaki. Integrals, conditional expectations and martingales of multivalued maps. J. Multivariate Anal., 7:149-182, 1977.

[20] T. Kato. Nonlinear semigroups and evolution equations. J. Math. Soc. Japan, 19:508520, 1967.

[21] T. Kato. Accretive operators and nonlinear evolutions in banach spaces. In Nonlinear Functional Analysis. Proc. Symp. Pure Math., 18, pages 138-161. Amer. Math. Soc, 1970.

[22] Y. Komura. Nonlinear semi-groups in hilbert space. J. Math. Soc. Japan, 19:493-507, 1967.

[23] D. Kravvaritis and N. S. Papagiorgiou. Multivalued perturbations of subdifferential type evolution equations in hilbert spaces. J. Differential Equations, 76:238-255, 1988.

[24] E. Michael. Continuous selections i. Ann. Math., 63:361-381, 1956.

[25] E. Mitidieri and I. Vrabie. Differential inclusions governed by nonconvex perturbations of m-accretive operators. Differential and Integral Equations, 2:515-531, 1989.

[26] C. Olech. Decomposability as a substitute for convexity. In Multifunctions and Integrands, pages 193-205. Lecture Notes in Mathematics 1091, Springer-Verlag, Berlin, 1984.

[27] M. Otani. On existence of strong solutions for $\frac{\partial u(t)}{\partial t}+\partial \phi^{1}(u(t))-\partial \phi^{2}(u(t)) \ni f(t)$. J. Fac. Sci. Univ. Tokyo, Sect I A, 24:575-605, 1977.

[28] M. Otani. Nonlinear evolution equations with time-dependent constraints. Advances in Mathematical Sciences and Applications Gakkotosho, Tokyo, 3:383-399, 1993/94.

[29] V. Staicu. Directionally continuous selections and nonconvex evolution inclusions. in preparation. 
[30] V. Staicu. On the solution sets to nonconvex differential inclusions of evolution type. In S. Hu and W. Chang, editors, Dynamical Systems and Differential Equations. Proceedings of the International Conference on Dynamical Systems and Differential Equations, Summer, 1996., pages 244-252. Southwest Missouri State University, Southwest Missouri State University, 1996.

[31] A. Syam. Contributions aux inclusions differentielles. $\mathrm{PhD}$ thesis, Universite Montpellier II, 1993.

[32] I. I. Vrabie. Compactness Methods for nonlinear evolutions. Longman, 1987. 